\title{
Codiseño del curso TIACE en la formación inicial docente de educación preescolar ante la contingencia de la COVID-19
}

\author{
Co-design of the ITAS course in the initial training of preschool education teachers in the face \\ of the COVID-19 contingency \\ iD Brenda Luz Colorado Aguilar \\ brendaluzcolorado@gmail.com \\ Benemérita Escuela Normal Veracruzana "Enrique C. Rébsamen” (México)
}

\begin{abstract}
Resumen
El objetivo del presente estudio se centró en valorar el co-diseño del curso, la Tecnología Informática Aplicada a los Centros Escolares (TIACE) en la formación inicial docente de la licenciatura en educación preescolar, ante la contingencia provocada por la COVID-19. La investigación de corte mixto y alcance descriptivo, se realizó en una escuela normal del estado de Veracruz, México. Se utilizó el cuestionario escala Likert aplicado a una muestra de 27 docentes en formación, así como la aplicación de 12 entrevistas semiestructuradas para el estudio fenomenológico hermenéutico. Se obtuvieron resultados favorables del co-diseño del curso en cuanto a las estrategias de organización y dinámica, aspectos pedagógicos, de contenido y tecnológicos. Con respecto a los factores que intervinieron para dar continuidad con las clases ante la pandemia, surgieron aspectos de estados de ánimo y contingencias familiares como factores a tomarse en cuenta en la modalidad de aprendizaje en línea.
\end{abstract}

Palabras clave: Co-diseño, formación docente, TIC, COVID-19.

\begin{abstract}
The objective of this study focused on evaluating the co-design of the course, Computer Technology Applied to School Centers (TIACE) in the initial teacher training of the degree in preschool education, in the face of the contingency caused by COVID-19. The research of mixed cut and descriptive scope was carried out in a normal school in the state of Veracruz, Mexico. The Likert scale questionnaire applied to a sample of 27 teachers in training was used, as well as the application of 12 semi-structured interviews for the hermeneutical phenomenological study. Favorable results were obtained from the co-design of the course in terms of organizational and dynamic strategies, pedagogical, content and technological aspects. With regard to the factors that intervened to continue with the classes in the face of the pandemic, aspects of moods and family contingencies emerged as factors to be taken into account in the online learning modality.
\end{abstract}

Keywords: Co-design, teacher training, ICT, COVID19. 


\section{INTRODUCCIÓN}

En el sistema educativo mexicano, las instancias formadoras de la mayor parte de los docentes que imparten clases en el nivel de educación básica (preescolar, primaria y secundaria) se les denomina escuelas normales. Históricamente estos centros educativos, han transitado por diferentes cambios y reformas educativas para enfrentar acontecimientos políticos, sociales, culturales y tecnológicos. Es sin duda que a inicios del año 2020 en el momento que la Organización Mundial de la Salud (OMS) declaró la contingencia a nivel mundial provocada por la COVID-19, se generó un suceso que ha transformado las prácticas pedagógicas de manera emergente, suscitado que las instituciones educativas estén enfrentando esta contingencia de manera heterogénea a nivel mundial, conforme sus posibilidades.

Tomando en cuenta que el paso de la escuela como lugar físico a un espacio virtual ante la pandemia es completamente nuevo (Delgado, 2020; Díaz, 2020; Martínez y Garcés, 2020; Plá, 2020; Suárez, 2020), en el contexto de las escuelas normales con la responsabilidad en la formación de profesionales de la educación, implica repensar la forma de abordar el magisterio, concordando con Díaz (2020) al argumentar que "la realidad está mostrando que es una circunstancia excepcional para aprender" (p. 26) y tomando en cuenta las palabras de Venegas et al. (2020), al expresar que el avance de las Tecnologías de la Información y la Comunicación (TIC) implica desafíos y ritmos para su incorporación educativa de manera constante.

Si bien es cierto que el compromiso institucional ante la contingencia, enfrenta decisiones tomadas en temas de inversión en infraestructura tecnológica, formación y actualización docente en el desarrollo de habilidades para el uso pedagógico de la tecnología, ente otros (Martínez y Garcés, 2020; Molina et al., 2012; Suárez, 2020); se espera que los esfuerzos y capacidad de resolución encuentren buen cause en el proceso educativo.

La Dirección General de Educación Superior para Profesionales de la Educación (DGESPE, 2020), para atender la nueva lógica de trabajo, ante la suspensión de actividades escolares por acuerdo de la SEP (2020); recomendó utilizar como apoyo al aprendizaje, plataformas y ecosistemas digitales que mejor se ajustaran al contexto en cuanto a las condiciones de conectividad y brecha digital. De modo que el Centro Virtual de Innovación Educativa de DEGESPE (CEVIE-DGESPE,2020), puso a disposición de la comunidad normalista, materiales y cursos para favorecer el perfil de egreso de los docentes en formación, dado que conforme el discurso de García et al. (2020) y Espinoza (2020) no se trata de trasladar las clases de lo presencial a la modalidad a distancia con la misma lógica educativa.

Por lo tanto, la modalidad de aprendizaje cambió de manera acelerada para dar continuidad a los cursos, tomando en cuenta que si bien es cierto que nos encontramos ante nuevas generaciones que están vinculadas con la tecnología, esto no significa que "necesariamente la emplean como un recurso de aprendizaje" (Díaz, 2020, p. 22). En este contexto es importante destacar a Jimoyiannis 
(2010) y Moore et al. (2011), cuando expresan que el verdadero aprendizaje con el uso de las TIC en la formación docente, se logra cuando se identifica como utilizarlas para gestionar información, innovar ideas y resolver problemáticas del mundo real; así también, el tomar en cuenta en palabras de Colorado (2014) a la usabilidad pedagógica de recursos y herramientas tecnológicas, cuando en su implementación se localiza significado didáctico para gestionar aprendizajes y finalmente enfatizar en la importancia de la articulación de elementos tecnológicos, pedagógicos y de contenido en los cursos (Baran et al., 2017; Koehler et al., 2013; Mishra y Koehler, 2006).

En este sentido, a través del aprendizaje en línea es posible promover la adquisición de competencias por parte de profesores y estudiantes, incorporando un elemento importante que es el tiempo real (Alfonso y Juanes, 2020). En esta modalidad el constructivismo social se hace presente cuando se establece mediación docente que promueve el trabajo colaborativo, motivando al estudio independiente y autorregulación por parte del estudiantado a través de la efectiva comunicación, colaboración y gestión de la información (Hernández, 2007). De tal forma que en palabras de Abreu (2020), "en tiempos de coronavirus la educación en línea debe dar su mejor respuesta a la crisis" (p. 1).

El tema del co-diseño, ha cobrado relevancia ante el escenario de la pandemia; considerando el ajuste a las prácticas educativas del entorno presencial al aprendizaje en línea, empleando estrategias para darle continuidad a los cursos ante una realidad de distanciamiento social, pérdida de la escuela como lugar de convivencia, socialización e intercambio de ideas para transitar a permanecer mayor tiempo de convivencia con la familia; impactando también en situaciones discordantes en cuanto al espacio físico, conectividad, estados de ánimo, entre otros. De acuerdo con el ITESM (2019) el co-diseño, formula estrategias para localizar soluciones de manera colaborativa a problemas; determinando la metodología que integra la construcción y diseño de la propuesta, el ambiente de aprendizaje, el contexto de los involucrados, forma de evaluación individual y/o colaborativa así como la modalidad; sea presencial o utilizando medios digitales.

Siguiendo a Westbroek et al. (2019), el co-diseño implica, estrategias para realizar adaptaciones y en ocasiones hasta verdaderas innovaciones a diseños curriculares, resultando que a nivel de aula, los docentes, realizan los ajustes necesarios para adaptar las situaciones de aprendizaje al contexto real de los estudiantes (Handelzalts, 2009; Severance et al., 2016; Voogt et al., 2005). De tal forma que se involucra al docente en su rol de facilitador y promotor de la participación y a los estudiantes colaborando con sus ideas para favorecer la experiencia de aprendizaje (ITESM, 2019). Cabe resaltar que en la formación de docentes, el co-diseño implica hacer frente a los ajustes necesarios ante la pandemia, porque conforme con González et al. (2020), trae consigo el "perder la presencialidad en las aulas y con ello la esencia de las prácticas de intervención" (p. 1), como parte de la identidad como profesionales de la educación.

En este contexto, el presente estudio se centró en la evaluación del co-diseño del curso que se imparte al interior de una escuela normal del estado de Veracruz en la licenciatura en educación preescolar denominado: La tecnología informática aplicada a los centros escolares (TIACE), que 
tiene como propósito: desarrollar conocimientos, actitudes y habilidades en el docente en formación acerca del uso de las TIC, "para adaptar su uso pedagógico en el contexto o ambiente educativo que se le presente" (SEP, 2012, p. 5).

Para realizar el co-diseño del curso TIACE al transitar de la modalidad presencial al aprendizaje en línea, se tomaron acuerdos de manera consensuada entre el docente formador y los docentes en formación, teniendo en consideración que este proceso de diseño implica, tanto a un "experto" como a "usuarios", para resolver un reto o problema en conjunto, desde su propia experiencia (ITESM, 2019, p.1). Por consiguiente, se establecieron las siguientes estrategias de trabajo:

- Utilizar la plataforma de aprendizaje google classroom para llevar acompañamiento, guía y retroalimentación del curso así como elegir en la práctica la plataforma de comunicación con mejor funcionalidad para las sesiones en línea.

- Tener en cuenta las condiciones en cuanto a disponibilidad de dispositivos digitales de los docentes en formación para realizar las sesiones de clase, actividades del curso y comunicación.

- Ajuste y reducción de contenidos del curso para seleccionar aquellos más significativos para generar de manera colaborativa, productos finales denominados "Planes de uso de herramientas y recursos digitales para apoyo de actividades didáctica en preescolar". Considerando que ante la imposibilidad de realizar las jornadas de intervención, se propondrían como materiales de apoyo para los docentes de las escuelas de práctica.

- Organizar equipos de trabajo colaborativo entre los docentes en formación para llevar a cabo las actividades del curso.

- Determinar un cronograma de sesiones de clase, entrega de actividades y asesorías personalizadas así como acuerdos acerca del proceso de evaluación.

- Permanecer en comunicación para conocer, factores personales y familiares que afectaran el desempeño del curso de los docentes en formación.

Para efectos del presente estudio se estableció como objetivo general:

- Valorar el co-diseño del curso TIACE en el aprendizaje en línea de los docentes en formación de la licenciatura en educación preescolar ante la contingencia provocada por la COVID-19.

Como objetivos específicos:

1. Valorar las estrategias para realizar el co-diseño de actividades educativas enriquecidas con las TIC para transitar de la modalidad presencial al aprendizaje en línea del curso TIACE.

2. Analizar los factores que intervienen en el aprendizaje de los docentes en formación ante la contingencia provocada por la COVID-19. 


\section{DISEÑO METODOLÓGICO}

Se realizó un estudio de corte mixto dado que era necesario abordar la problemática desde una perspectiva más amplia y profunda que permitiera cuantificar y cualificar el fenómeno; con alcance exploratorio-descriptivo, para lograr mayor familiaridad con la problemática y proseguir con su descripción, con el fin de medir y recabar información sobre las variables (Hernández y Mendoza, 2018).

De tal forma que el estudio se realizó en una escuela normal pública, ubicada en una zona urbana de la capital del estado de Veracruz, México. Los estudiantes de 5 semestre de la licenciatura en educación preescolar fueron la población objeto de estudio con una muestra no probabilística porque los criterios para elegirla no dependieron de la probabilidad sino de las características de la propia investigación (Hernández y Mendoza, 2018; Stockemer, 2020). Es así que la muestra estuvo constituida por 26 docentes del sexo femenino y 1 del sexo masculino, con edades entre los 18 a los 24 años; con la exigencia de que pertenecieran al 50 semestre y fueran alumnos del curso TIACE de la licenciatura en educación preescolar.

Para el análisis cualitativo participaron 12 de los docentes en formación con los mismos criterios de inclusión de la muestra cuantitativa. El estudio se llevó a cabo a través del diseño fenomenológico hermenéutico "pues conlleva a reflexionar en profundidad acerca de las experiencias cotidianas, encontrar el significado de estas experiencias de modo único en cada individuo con el fin de poseer capacidad de tomar acciones que lleven a mejorar la práctica pedagógica" (Fuster, 2019, p.206).

El análisis de datos para la parte cuantitativa, se realizó con el software IBM SPSS, Statistics Base V.22.0 y con el programa para análisis de datos cualitativos MAXQDA Analitics pro V.2020.0.8. En el enfoque cuantitativo se aplicaron medidas de tendencia central y variabilidad atendiendo al alcance del estudio. El análisis cualitativo se realizó conforme la teoría fundamentada, que inicia categorizando los datos de manera abierta, posteriormente se atiende al modelo de interrelaciones conforme la codificación axial para llegar a la organización de las unidades de significado a través de la codificación selectiva (Kuckartz, 2014). De tal forma que las categorías van emergiendo a partir del examen de los datos, para ilustrar con ejemplos la forma en la cual se caracterizan (Glaser y Strauss, 2017).

\subsection{Instrumentos}

Se aplicaron 2 instrumentos de recolección de datos, el primero: un cuestionario con 25 ítems escala Likert que fue validado por juicio de 5 expertos elegidos por su formación en posgrado y dominio en el área de las TIC así como por su conocimiento y experiencia en el diseño y co-diseño de planes y programas de estudio del sistema mexicano de educación normal. 
La validez por juicio de expertos fue calculada a través del coeficiente $V$ de Aiken (Boluarte $y$ Tamari, 2017). Se tomaron en cuenta criterios de claridad, coherencia, relevancia y suficiencia. Dado que los valores que arroja el coeficiente se ubican entre 0 y 1 , entre más cercano o igual sea el valor a 1, corresponde a mayor acuerdo entre los jueces sobre la validez de contenido de los items, que llevó a suprimir o modificar aquellos que no fueran estadísticamente significativos. La confiabilidad se obtuvo mediante el Alpha de Cronbach con un valor de 0.845 .

El cuestionario con escala tipo Likert que consideró los 3 primeros ítems con información demográfica, se estructuró en las siguientes 4 dimensiones:

1. Organización y dinámica del curso. Organización del curso conforme a la dinámica atendiendo tanto el rol del docente formador como de los docentes en formación.

2. Aspectos pedagógicos y de contenido. Actividades educativas con estrategias de enseñanza específica y temas del curso completamente relacionadas con su propósito. (Aguilar y Cabero, 2015).

3. Aspectos tecnológicos. Herramientas y recursos digitales implementados en la modalidad en línea para favorecer el aprendizaje colaborativo de los docentes en formación.

4. Factores para el aprendizaje ante la contingencia por la COVID-19. Situaciones de estado de ánimo entendida como respuestas emocionales de las personas (Urzúa et al. 2020), ante el impacto de la pandemia, así como las condiciones del cambio de modalidad del curso (de presencial a aprendizaje en línea) en el entorno familiar.

Como segundo instrumento, se aplicó la entrevista semiestructurada sometida a validación de expertos diseñada a partir de una tabla de categorización de variables para el diseño de la guía de preguntas.

\section{RESULTADOS}

Se organizó la presentación de resultados a través de las dimensiones del cuestionario aplicado para el estudio cuantitativo que se complementó con los datos obtenidos de las entrevistas atendiendo al diseño fenomenológico hermenéutico y de teoría fundamentada. Las abreviaturas que se utilizaron de H y RD corresponden a Herramientas y Recursos Digitales y DF (n) se refiere a Docentes en Formación especificando el número de participante en el estudio.

\subsection{Estrategias para el co-diseño del curso TIACE. Formas de trabajo llevadas a cabo, a partir del el co-diseño del curso.}




\subsubsection{Organización y dinámica del curso.}

Las medidas de tendencia central y variabilidad reflejaron valores favorables como puede observarse en la Tabla 1, con respecto a las opiniones del docente formador y docentes en formación.

Tabla 1. Organización y dinámica del curso

\begin{tabular}{|l|c|c|}
\hline Dimensiones & Media & Desviación estándar \\
\hline \multicolumn{2}{|l|}{ Rol docente formador: } \\
\hline Manejo del grupo en las sesiones & 4.92593 & 0.26688 \\
\hline Utilización de H y RD para dinámica sesiones & 4.81481 & 0.48334 \\
\hline Sesiones de clase por equipos & 4.81481 & 0.39585 \\
\hline Procesos de evaluación & 4.40741 & 0.57239 \\
\hline Rol docentes en formación: & \multicolumn{2}{|l}{} \\
\hline Dinámica en equipos de trabajo & 4.22222 & 0.84732 \\
\hline Organización de tiempo & 3.74074 & 0.71213 \\
\hline Organización en el espacio físico-hogar & 4.07407 & 1.141050 \\
\hline
\end{tabular}

En cuanto al rol de los docentes en formación (Tabla 2), se denota mayor dispersión en las dimensiones de: Dinámica en equipos de trabajo, Organización de tiempo y Organización del espacio físico-hogar; para llevar a cabo las actividades escolares en la modalidad en línea. 
Tabla 2. Rol docentes en formación

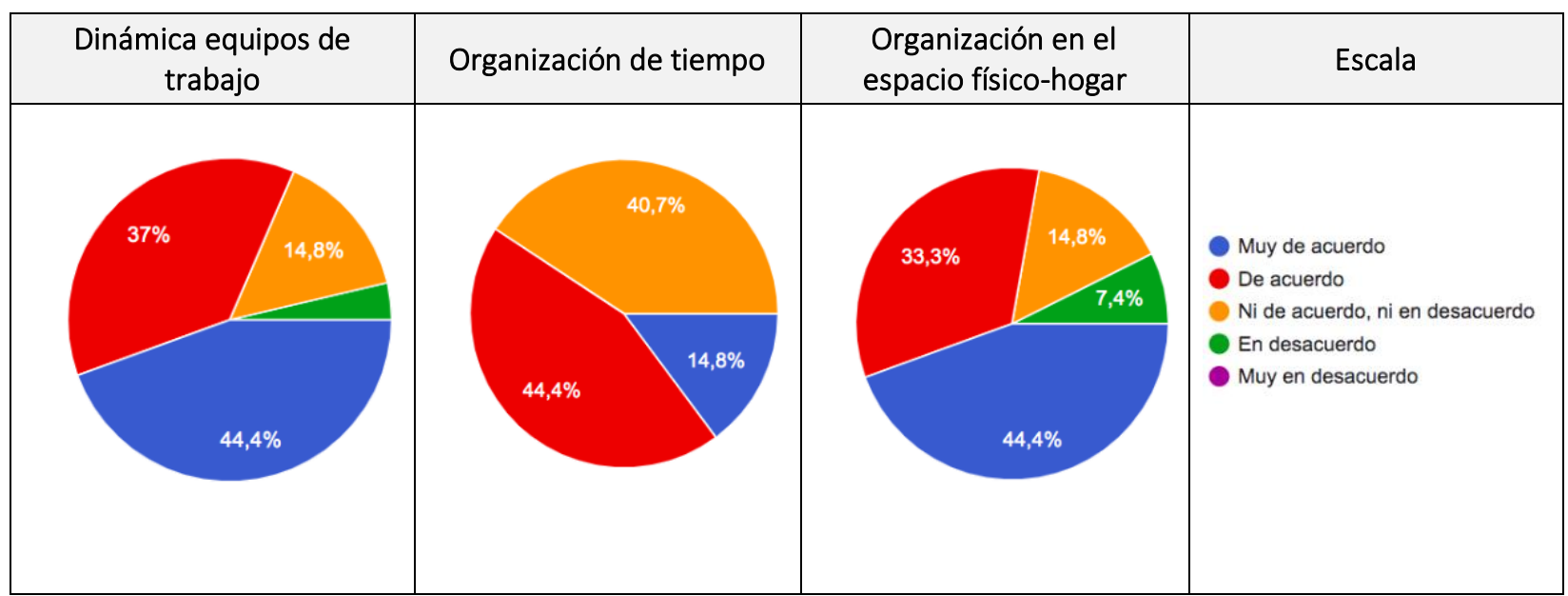

Cabe destacar que los procesos de evaluación formativa a lo largo del semestre y de evaluación sumativa con la valoración del diseño de un producto final; resultaron con tendencia favorable en opinión de los docentes en formación: "utilizamos recursos digitales aprendidos en el curso para diseñar el producto final con relación a los aprendizajes esperados del preescolar, fue grata la experiencia de trabajo en equipo" (DF, 5). Dichos productos consistieron en "Planes de uso de herramientas y recursos digitales para apoyo de actividades didáctica en preescolar que fueron propuestos como materiales de apoyo para los docentes de las escuelas de práctica" $(D F, 1)$.

En el estudio cualitativo se profundizó en la categoría de Organización y dinámica del curso, destacando con mayor peso de discusión las subcategorías que pueden observarse en la Figura. 1, con las rayas más gruesas y el número al lado derecho que muestra la frecuencia de codificaciones: comunicación, acompañamiento y empatía, así como en organización y dinámica de las sesiones por equipo. Cabe mencionar que las sesiones en las plataforma de videoconferencia, se realizaron por equipos de trabajo, para mejorar la interacción y comunicación; lo que dio como resultado opiniones favorables. 


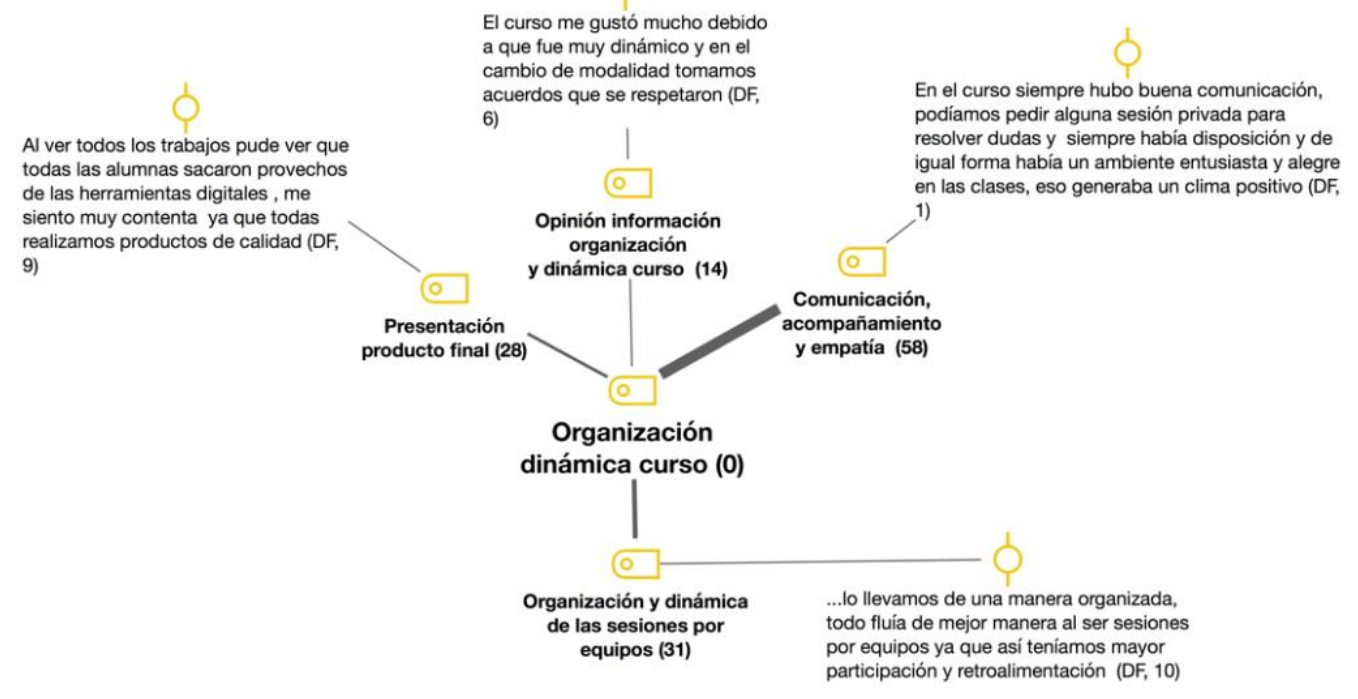

Figura 1. Organización y dinámica del curso.

\subsubsection{Aspectos pedagógicos y de contenido.}

Los valores de las medidas de tendencia central y variabilidad de esta dimensión estuvieron por arriba de la mediana de los datos como puede observarse en la Tabla 3. Es así que los docentes en formación tuvieron opiniones positivas en cuanto al aprendizaje del contenido del curso TIACE, reportando haber incrementado sus conocimientos sobre nuevas herramientas y recursos digitales para utilizar en el nivel de preescolar, así como aquellas que pueden elegir para favorecer el desarrollo de aprendizajes esperados de los preescolares. También fue favorable en cuanto al trabajo colaborativo realizado en equipos de trabajo y con respecto al aprendizaje significativo obtenido en el curso.

Tabla 3. Aspecto pedagógico y de contenido

\begin{tabular}{|l|c|c|}
\hline Dimensiones & Media & $\begin{array}{c}\text { Desviación } \\
\text { estándar }\end{array}$ \\
\hline Aprendizaje del contenido del curso & 4.81481 & 0.48334 \\
\hline $\begin{array}{l}\text { Aprendizaje nuevos H y RD para nivel de } \\
\text { preescolar }\end{array}$ & 4.96296 & 0.19245 \\
\hline $\begin{array}{l}\text { Identificación de H y RD para desarrollo } \\
\text { de aprendizaje esperados }\end{array}$ & 4.74074 & 0.44658 \\
\hline Trabajo colaborativo & 4.70370 & 0.46532 \\
\hline Aprendizaje significativo en el curso & 4.88889 & 0.32026 \\
\hline
\end{tabular}


En el análisis cualitativo se identificó la categoría de Aspecto pedagógico y de contenido. Como subcategorías: Aprendizaje en tipo de $H$ y RD, Factores para utilizar H y RD y Trabajo colaborativo, que se explican a continuación:

- Aprendizaje tipo de $H$ y RD.

En esta subcategoría se recuperó la frecuencia de códigos acerca del tipo de herramienta y recursos digitales que los docentes en formación opinaron haber aprendido a utilizar en el curso (Figura 2).

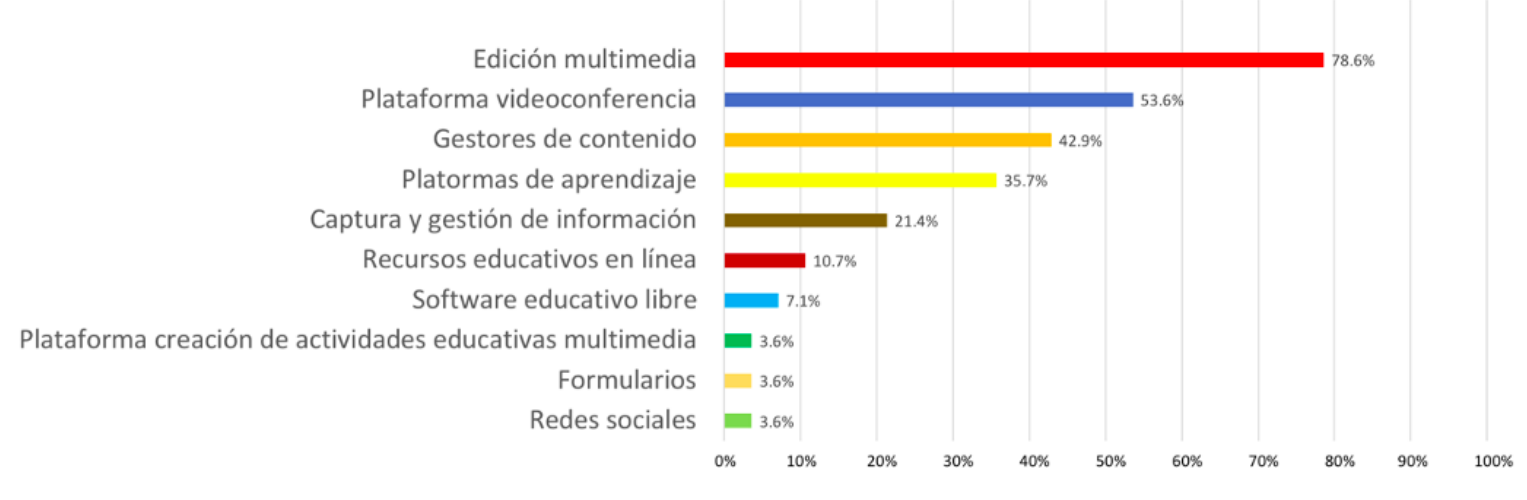

Figura 2. Opinión sobre aprendizaje en el uso de herramientas y recursos digitales.

\section{- Factores para utilizar H y RD.}

Subcategoría que integró las opiniones de los docentes en formación sobre aquellos factores que deben tomarse en cuenta para el uso de herramientas y recursos digitales en el nivel de preescolar (Tabla 4).

Tabla 4. Factores para implementar herramientas y recursos digitales con preescolares

\begin{tabular}{|l|l|}
\hline \multicolumn{2}{|c|}{ Subcategoría: Factores a considerar para implementar H y RD } \\
\hline Enfoque pedagógico & $\begin{array}{l}\text { “..no se trata solo de llevar una laptop al salón y ponerles un } \\
\text { video a los preescolares, sino de desarrollar su cognición } \\
\text { mediante la experimentación con juegos, cuentos y } \\
\text { actividades"(DF, 11). }\end{array}$ \\
\hline $\begin{array}{l}\text { Contexto y } \\
\text { característica de } \\
\text { preescolares }\end{array}$ & $\begin{array}{l}\text { “..nosotros como docentes debemos involucrar a nuestros } \\
\text { alumnos al uso de la tecnología, tomando como ejemplo } \\
\text { problemas de la vida cotidiana e irlos involucrando conforme sus } \\
\text { características"(DF, 12). }\end{array}$ \\
\hline
\end{tabular}




\begin{tabular}{|l|l|}
\hline \multicolumn{2}{|c|}{ Subcategoría: Factores a considerar para implementar H y RD } \\
\hline $\begin{array}{l}\text { Conocimiento y } \\
\text { habilidad uso de H y RD }\end{array}$ & $\begin{array}{l}\text { "en el curso avanzamos en nuestras habiliadades para usar los } \\
\text { recursos digitales y el conocimiento para utilizarlas en el nivel } \\
\text { preescolar" (DF, 7). }\end{array}$ \\
\hline $\begin{array}{l}\text { Desarrollo de } \\
\text { aprendizajes esperado }\end{array}$ & $\begin{array}{l}\text { "tener en cuenta los aprendizajes esperados a desarrollar en los } \\
\text { preescolares con el uso de TIC" (DF, 6). }\end{array}$ \\
\hline Modalidad de trabajo & $\begin{array}{l}\text { "Al final de este curso pude darme cuenta cómo puedo utilizar la } \\
\text { tecnología para integrarla con los preescolares en la nueva } \\
\text { modalidad de aprendizaje en línea que ahora conocemos" (DF, 8). }\end{array}$ \\
\hline
\end{tabular}

\section{- Trabajo colaborativo.}

La categoría de trabajo colaborativo que los docentes en formación realizaron organizados en equipos de trabajo, se dividió en tres subcategorías: favorable, mejorable y no favorable; resultando la primera con mayor satisfacción conforme se observa en la Figura 3, con la raya de mayor grosor. Con menor peso, resultaron las opiniones de mejorable por problemas de conexión y no favorable por falta de organización en el equipo de trabajo.

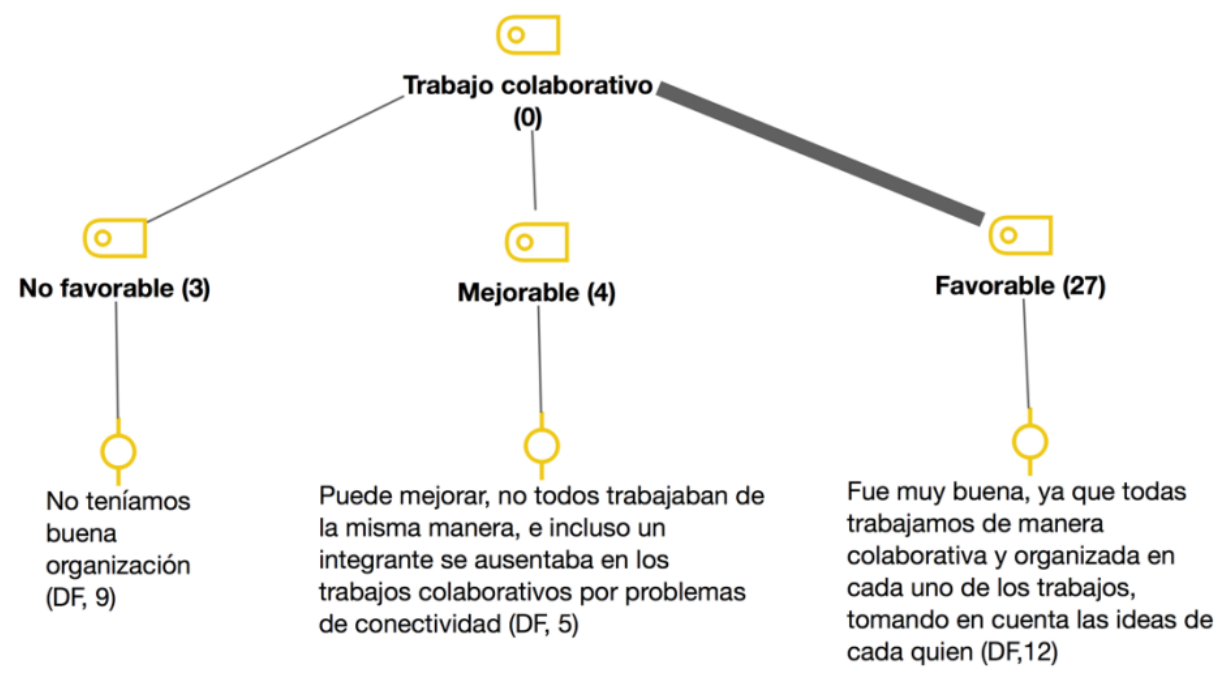

Figura 3. Trabajo colaborativo. 


\subsubsection{Aspectos tecnológicos.}

Se utilizó la plataforma de aprendizaje Google Classroom para gestionar el curso y para las sesiones de clase se emplearon diferentes plataformas de videoconferencia. En cuanto a los medios para establecer comunicación se dispusieron aquellos que tenían al alcance los docentes en formación. En esta dimensión se presentan los resultados acerca de las condiciones de conectividad, funcionalidad y manejo de dichas herramientas para llevar a cabo el curso.

En el análisis cuantitativo los valores que ocurrieron con mayor frecuencia conforme la escala de Likert fueron: "De acuerdo", con medidas de tendencia central y variabilidad favorables como puede observarse en la Tabla 5.

Tabla 5. Plataformas de trabajo en línea

\begin{tabular}{|l|c|c|}
\hline Dimensiones & Media & $\begin{array}{c}\text { Desviación } \\
\text { estándar }\end{array}$ \\
\hline $\begin{array}{l}\text { Conectividad sesiones de clase y } \\
\text { Classroom }\end{array}$ & 4.11111 & 0.89156 \\
\hline $\begin{array}{l}\text { Manejo y funcionalidad plataformas de } \\
\text { comunicación sesiones en línea }\end{array}$ & 4.40741 & 0.57239 \\
\hline Manejo y funcionalidad classrroom & 4.74074 & 0.52569 \\
\hline
\end{tabular}

En cuanto a las plataformas de videoconferencia que consideraron de mayor funcionalidad para las sesiones de clase resultaron Zoom y Google Meet. El valor de la moda representa las mejor evaluadas con mayor frecuencia (Figura 4).

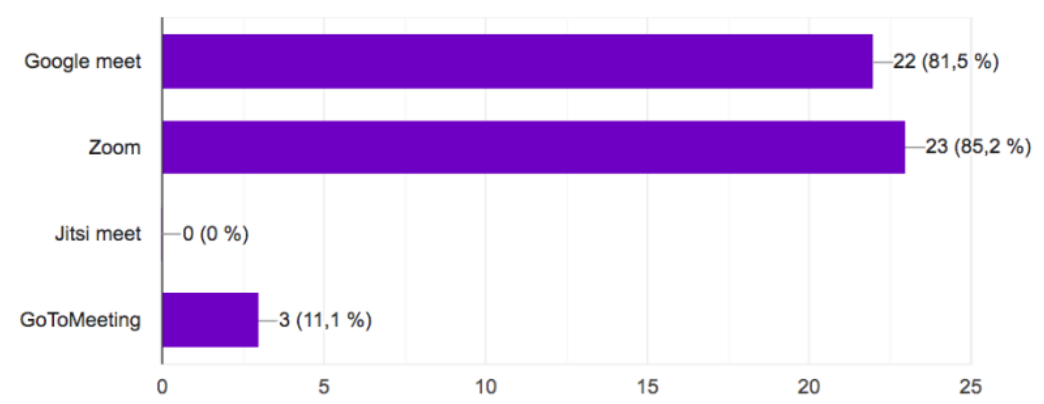

Figura 4. Plataformas de videoconferencia conforme su funcionalidad.

Los medios de comunicación que se utilizaron con mayor frecuencia para establecer comunicación entre los docentes en formación y el docente formador fue el WhatsApp, a través de mensajes en grupo o de forma privada (Figura 5). 


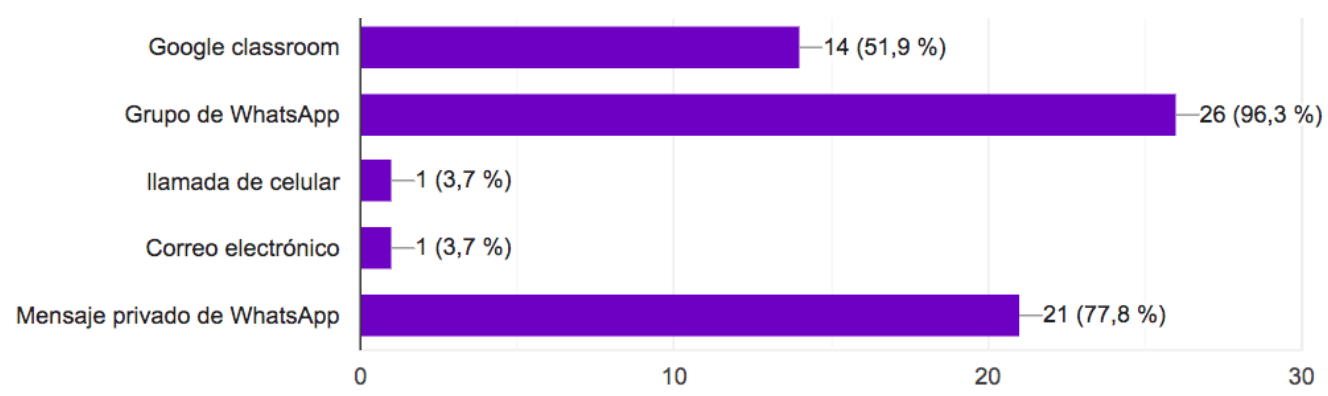

Figura 5. Opinión sobre aprendizaje en el uso de herramientas y recursos digitales.

También se midió el tipo de medio para establecer comunicación entre los compañeros de equipo, reportando el grupo de WhatsApp con mayor frecuencia (Figura 6).

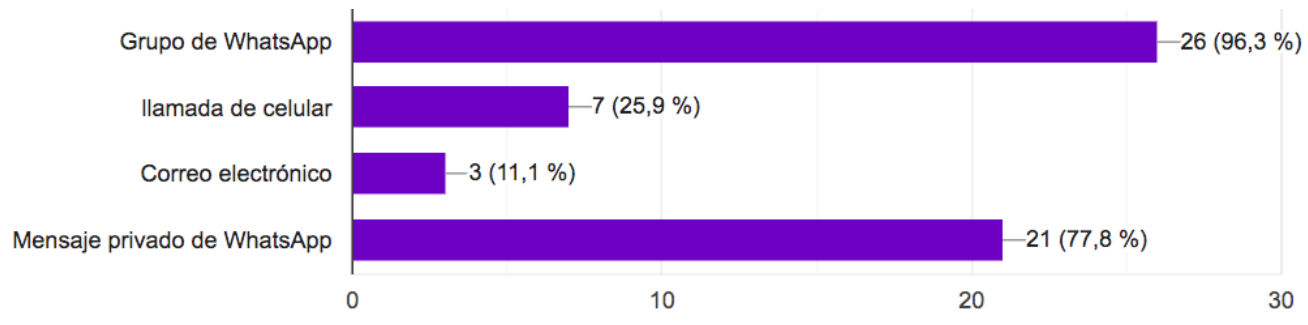

Figura 6. Opinión sobre aprendizaje en el uso de herramientas y recursos digitales.

En el estudio cualitativo se identificó la categoría de aspecto tecnológico, al transitar de las sesiones presenciales a sesiones en línea, destacando como subcategoría: Cambio de modalidad, que a su vez se subdividió en subcategorías de menor nivel: Experiencia de aprendizaje favorable, Experiencia de uso $H$ y RD, con peso más alto, conforme con la raya de mayor grosor. En cuanto a las rayas de menor grosor fueron: Costumbre en la modalidad presencial y la incomodidad por tener las Sesiones en el entorno familiar así como Cansancio ocular con menor peso de discusión (Figura 7). 


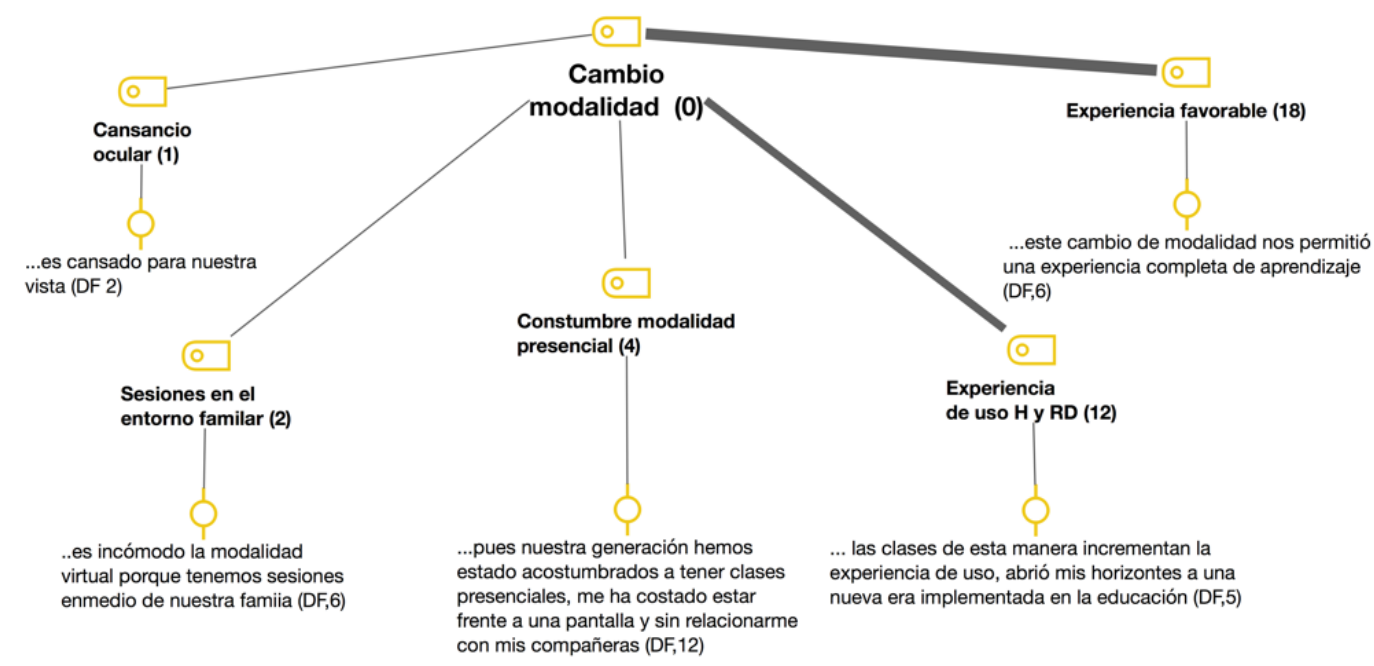

Figura 7. Cambio de modalidad.

\subsection{Factores que intervinieron durante el desarrollo del curso.}

En cuanto a los factores que intervinieron en el aprendizaje de los docentes en formación ante la contingencia provocada por la COVID-19, los estados de ánimo manifestados durante el tiempo en el cual se desarrolló el curso resultaron un aspecto importante. Conforme el estudio cuantitativo el estrés, incertidumbre y cansancio tuvieron mayor presencia (Figura 8), que se complementó con el estudio cualitativo destacando las subcategorías: Estresada/o: "Me estresé al imaginar como seria la manera de evaluar los trabajos que se solicitarían y sentí que esto podría ocasionar que me fuera mal dentro de los cursos por no poder contar con los recursos necesarios y cómo podría apoyar a mis hermanos para que no se sintieran perdidos como yo" (DF,2), con Sentimientos de incertidumbre: "Nunca había pensado que me tocaría vivir una época así, donde tuviera que permanecer en casa con la incertidumbre de lo que pasaría" (DF, 8).

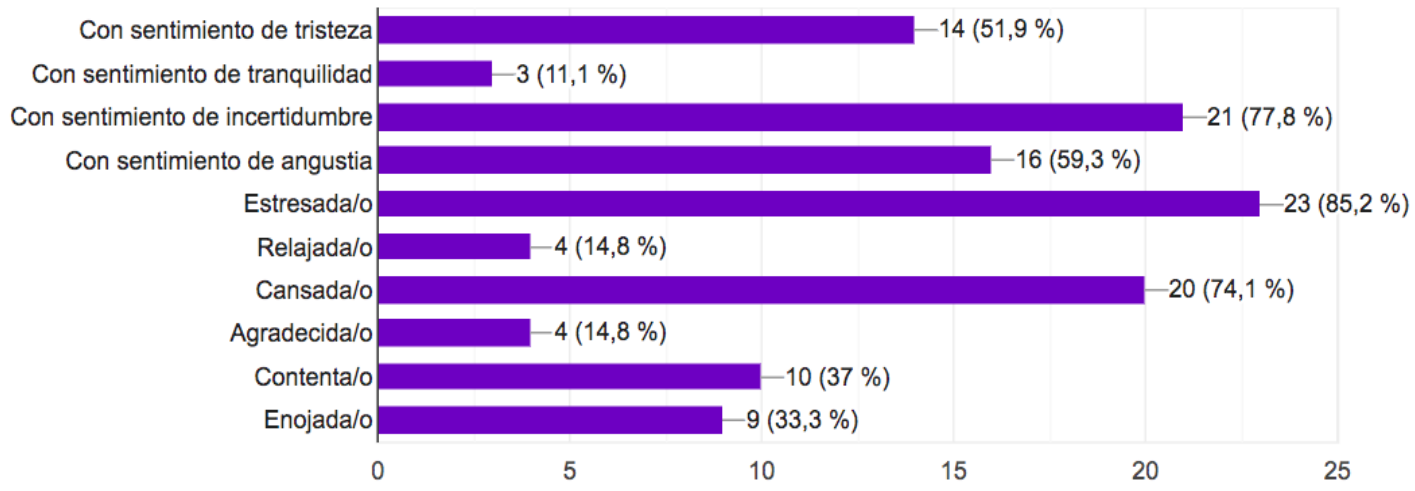

Figura 8. Categoría información, organización, dinámica curso. 
Dentro de la categoría Contingencias familiares se identificaron subcategorías, que caracterizaron la realidad que se hizo presente en el contexto de los docentes en formación.

- Contingencias familiares: La situación de salud fue un factor importante durante las clases, que se expresó dentro de tres subcategorías: Muerte de familiar, Familiar enfermo de COVID-19 e Inserción al campo laboral por situación económica complicada: "me pesa estudiar ante la muerte de mi familiar" (DF,4), "tuve que comenzar a trabajar porque se complicó la situación económica en mi casa" (DF,6), "tengo un familiar enfermo de COVID y me cuesta concentrarme" (DF, 9).

\section{DISCUSIÓN Y CONCLUSIONES}

El estudio para valorar el co-diseño del curso TIACE en el aprendizaje en línea de los docentes en formación de la licenciatura en educación preescolar, ante la contingencia provocada por la COVID19, resultó favorable con respecto a las medidas estadísticas aplicadas y el estudio fenomenológico hermenéutico con base en la teoría fundamentada para la parte cualitativa.

Como parte de las estrategias empleadas para el co-diseño del curso, la organización y dinámica resultaron positivas ante las condiciones de transitar de manera emergente, de las aulas al hogar para dar continuidad con las clases en línea y enfrentar los factores que se hicieron presentes ante la contingencia.

En los resultados cuantitativos de las dimensiones del rol de los docentes en formación se detectó mayor dispersión y diferencia en la tendencia de los datos, que si bien resultaron favorables, es importante tomar en cuenta que el aprendizaje en el línea implica considerar factores para favorecer el aprendizaje, como: problemas de conectividad que afectan la dinámica de trabajo entre los integrantes de los equipos, la organización personal del tiempo y del espacio físico para realizar las actividades escolares en el hogar, ya que conforme con García et al. (2020) y Espinoza (2020), es diferente estar en la escuela con dedicación al proceso de formación que estar en el hogar con otro tipo de actividades y jornadas que cumplir.

Se destaca en el estudio cualitativo que la comunicación, acompañamiento y empatía del docente formador, así como la organización y dinámica de las sesiones por equipo resultaron positivas; siguiendo el discurso de Espinoza (2020), al expresar que el ambiente de tranquilidad y apoyo del docente formador favorece el aprendizaje porque se establece el vínculo con lo emocional; dada la situación actual que en palabras de García et al. (2020), se viven "momentos extremadamente complicados, a los que nunca nos habíamos enfrentado con anterioridad, con toda la población confinada en sus casas, luchando contra un virus, en un estado de alerta" (p. 83).

En lo que respecta a los aspectos pedagógico, de contenido y tecnológico se detectaron evidencias favorables al transitar de la modalidad presencial al aprendizaje en línea, que conforme Baran et 
al. (2017), la articulación de estos tres elementos es una lógica importante de considerar en la formación inicial docente como una estrategia para desarrollar las habilidades para el uso eficiente de la tecnología. De tal forma que ante la nueva modalidad de aprendizaje que surgió de manera emergente, el co-diseño del curso TIACE favoreció aspectos importantes conforme los resultados del estudio, dado que los docentes en formación destacaron los aspectos pedagógicos, de contenido y tecnológicos al otorgarle valoraciones por arriba de la mediana al aprendizaje significativo de los contenidos del curso, así como al valor didáctico que localizaron en las herramientas y recursos digitales (Colorado, 2014), como funcionales para el nivel de preescolar; lo que trae consigo "ir más allá de los enfoques simplificados que tratan la tecnología como un "complemento" para centrarse en cambio, y de una manera más ecológica, en las conexiones entre tecnología, contenido y pedagogía" (Koehler et al., 2013, p. 18).

Así también se da muestra del trabajo colaborativo de los docentes en formación con los productos finales realizados (https://sites.google.com/view/tiacepreesco/inicio), en los cuales integraron herramientas y recursos para diseñar materiales digitales de apoyo para los docentes de las escuelas de práctica; dado que no les fue posible realizar las jornadas de intervención por la pandemia, suscitando una situación complicada, dado que conforme con González et al. (2020), resalta la importancia de estas jornadas de práctica profesional porque "encuentran en este periodo un sentido a la profesión docente, despertando en ellos una actitud reflexiva y crítica sobre qué hacen y cómo lo hacen, y por tanto, conformando su identidad docente" (p. 1).

Resulta importante la reflexión constructiva de los docentes en formación con respecto a los elementos que deben tomarse en cuenta a la hora de introducir el uso de las TIC en cuanto al enfoque pedagógico, contexto y característica de los alumnos, desarrollo de aprendizajes esperados y modalidad de trabajo en el nivel de preescolar; tomando en cuenta el discurso de Venegas et al. (2020), quienes manifiestan la importancia de asumir por parte de las instituciones educativas el compromiso "sobre la formación, soporte y asesoramiento técnico-pedagógico y/o técnico-instruccional en el uso de TIC para los procesos de enseñanza- aprendizaje" (p. 40), porque son elementos indispensable para la incorporación de la tecnología en la práctica educativa.

Las plataformas que resultaron con opiniones positivas para establecer mediación pedagógica fueron Google Classroom, Google Meet y Zoom. Para la comunicación entre pares y con el docente formador destacó el grupo de whats App; aspecto a tomar en cuenta dado que, en la formación de docentes "el tratamiento que se haga de la información, la comprensión, análisis y producción de mensajes, vendrá muy determinada por la formación y enfoque metodológico que reciban durante su formación inicial" (Molina et. al, 2012, p.4). Es así que en tiempos de pandemia, los docentes en formación favorecieron su aprendizaje en los aspectos mencionados para el uso de mensajería, mismos que anteriormente no habían distinguido para establecer comunicación significativa con sus compañeros de grupo y con el docente formador; así como el identificar plataformas de aprendizaje y comunicación para facilitar la gestión de su aprendizaje. 
Cabe mencionar que dentro de los factores para el aprendizaje ante la contingencia por la COVID19, los estados de ánimo que mayor se manifestaron fueron estrés, incertidumbre y cansancio ante la situación de confinamiento, así como las contingencias familiares en agravio de la salud y muerte de familiares, para proseguir con su formación. Cabe mencionar que a partir de estos tiempos de pandemia impera "crear nuevos paradigmas psicoeducativos para la educación; marcos que permitan tomar en cuenta que el motor del comportamiento humano son sus afectos y sus emociones, elementos que, en las aulas de todos los niveles educativos, se han olvidado" (Delgado, 2020 , p. 188) y que actualmente deben tomarse en cuenta con mayor peso en el diseño de los programas educativos.

Si bien se reconoce plenamente el valor de la educación presencial, se puede concluir que el codiseño del curso a través de las estrategias de trabajo empleadas, reportó resultados favorables, tomando en cuenta que "el co-diseño representa un enfoque emergente dentro de las ciencias del aprendizaje para producir innovaciones más utilizables y para expandir la agencia de los maestros en el proceso de mejorar la enseñanza y el aprendizaje" (Severance et al.,2016, p.3).

En este sentido, el discurso de Abreu (2020) cobra relevancia al expresar que la educación en línea muestra su potencial, siempre que sea bien gestionada para el logro de los aprendizajes. Así como a Espinoza (2020) al enfatizar en la importancia del acompañamiento del docente formador estableciendo un vínculo entre la parte emocional y el aprendizaje, condiciones clave para la situación actual de contingencia ante la pandemia.

\section{REFERENCIAS}

Aguilar, S. y Cabero. J. (2015). La formación del profesorado en Tic: Modelo TPACK (Conocimiento Tecnológico, Pedagógico y de Contenido). Sevilla: Secretariado de Recursos Audiovisuales y Nuevas Tecnologías de la Universidad de Sevilla. Píxel-Bit. Revista de Medios y Educación, (46), 253-254. https://bit.ly/3kNg1H6

Abreu, J.L. (2020). Tiempos de Coronavirus: La Educación en Línea como Respuesta a la Crisis. Daena: International Journal of Good Conscience, 15(1)1-15. https://bit.ly/3aM9LM6

Alfonso, Y. y Juanes, B. Y. (2020). Estrategia de implementación del aprendizaje en línea en el Centro de Formación en Docencia Universitaria. Revista Metropolitana de Ciencias Aplicadas, 3(2), 148-158. https://bit.ly/3nXcDez

Baran, E. Canbazoglu, S., Albayrak, A. y Tondeur, J. (2017). Investigating the impact of teacher education strategies on preservice teachers' TPACK. British Journal of Educational Technology, 50, (1), 357-370. https://doi.org/10.1111/bjet.12565 
Boluarte, A. y Tamari, K. (2017). Validez de contenido y confiabilidad inter-observadores de Escala Integral Calidad de Vida. Revista de Psicología, 35 (2). https://doi.org/10.18800/psico.201702.009

CEVIE-DGESPE (abril de 2020). Recomendaciones adicionales para implementar la Estrategia de trabajo en Línea. Secretaría de Educación. https://bit.ly/365zU7V

Colorado, B.L. (2014). Usabilidad de las Tecnologías y Información y Comunicación (TIC) en la práctica Educativa. (Tesis de doctorado, Universidad Veracruzana) https://bit.ly/3q5EScK

Delgado, G. (2020). Igualdad educativa y postpandemia. En Educación y Pandemia. Una visión académica. UNAM. https://bit.ly/35MWS3C

DGESPE (1 de junio 2020). Orientaciones autoridades de Educación Normal. [Archivo de Vídeo]. Youtube. https://bit.ly/3fzt8L3

Díaz, A. (2020). La escuela ausente, la necesidad de replantear su significado. En Educación y Pandemia. Una visión académica. UNAM. https://bit.ly/3319JgO

Espinoza, V. A. (5 de agosto 2020). Lecciones de la pandemia y el futuro de la formación docente Centro Regional de Formación Docente e Investigación Educativa. [Ponencia]. https://bit.ly/3mb07Yp

Fuster, D.E. (2019). Investigación cualitativa: Método fenomenológico. Hermenéutico. Propósitos y Representaciones, 7, (1), 201 - 229. http://dx.doi.org/10.20511/pyr2019.v7n1.267

García, N., Rivero, M.L. y Ricis, J. (2020). Brecha digital en tiempos de COVID-19. Revista Educativa Hekademos, 28, 76-85. https://bit.ly/2DPIcWV

Glaser, B.G. y Strauss, A. L. (2017). The discovery of grounded theory. Strategies for qualitative research. Routledge Taylor \& Francis Group.

González, G., Barba, R.A., Bores, D. y Gallego, V. (2020). Learning to be a teacher without being in the Classroom: COVID-19 as a threat to the professional development of future teachers. International and Multidisciplinary Journal of Social Sciences, 9 (2), 152-177. http://dx.doi.org/10.17583/rimcis.2020.5783

Handelzalts, A. (2009). Collaborative curriculum development in teacher design teams (tesis doctoral). https://doi.org/10.3990/1.9789036528634.

Hernández, S. C. (2007). El constructivismo social como apoyo en el aprendizaje en línea. Apertura, 7(7),46-62. https://bit.ly/3aa5W3d

Hernández, R. y Mendoza, Ch. P. (2018). Metodología de la investigación. Las rutas cuantitativas, cualitativa y mixta. McGraw Hill. 
ITESM (2019). Aprendizaje activo 4.0 Co-diseño. [Imagen]. Innovacion educativa en el Tecnológico de Monterrey. https://bit.ly/3nRz6ts

Jimoyiannis, A. (2010). Designing and implementing an integrated technological pedagogical science knowledge framework for science teachers professional development. Computers \& Education, 55, 1259-1269. https://dl.acm.org/doi/10.1016/j.compedu.2010.05.022

Koehler, M. J., Mishra, P. y Cain, W. (2013). What is Technological Pedagogical Content Knowledge (TPACK)? The Journal of Education, 193, (3), 13-19. https://bit.ly/37alDoL

Kuckartz, U. (2014). Qualitative Text Analysis. SAGE Publications Ltd.

Martínez, J. y Garcés, J. (2020). Competencias digitales docentes y el reto de la educación virtual derivado de la covid-19. Educación y Humanismo, 22(39), 1-16. https://doi.org/10.17081/eduhum.22.39.4114

Molina, M. D., Pérez, A. y Antiñolo, J. L. (2012). Las TIC en la formación inicial y en la formación permanente del profesorado de infantil y primaria. Edutec. Revista Electrónica de Tecnología Educativa, (41), 1-23. https://doi.org/10.21556/edutec.2012.41.353

Moore, J.L., Dickson, C. y Galyenb, K. (2011). e-Learning, online learning, and distance learning environments: Are they the same? Internet and Higher Education, 14(2), 129-135. https://doi.org/10.1016/j.iheduc.2010.10.001

Mishra, P. y Koehler, M. J. (2006). Technological pedagogical content knowledge: A framework for teacher knowledge. Teachers College Record, 108(6), 1017-1054. https://doi.org/10.1111/j.1467-9620.2006.00684.x

Plá, S. (2020). La escuela ausente, la necesidad de replantear su significado. En Educación y Pandemia. Una visión académica. UNAM. https://bit.ly/2ILMdOc

SEP (2020, 16 marzo). ACUERDO número 02/03/20 por el que se suspenden las clases en las escuelas de educación preescolar, primaria, secundaria, normal y demás para la formación de maestros de educación básica del Sistema Educativo Nacional, así como aquellas de los tipos medio superior y superior dependientes de la Secretaría de Educación Pública. Diario Oficial de la Federación. https://bit.ly/33zBGNV

SEP (2012). Planes de estudio. Lic. en Educación Preescolar (plan 2012). DGESPE. https://bit.ly/2J1u6ny

Severance, S., Penuel, W. R., Sumner, T. y Leary, H. (2016). Organizing for teacher agency in curricular co-design. Journal of the Learning Sciences, 25(4), 531-564. https://doi.org/10.1080/10508406.2016.1207541 
Stockemer, D. (2020). Quantitative Methods for the Social Sciences. A practical introduction with examples in SPSS and Stata. Springer.

Suárez, N. (2020). Formación docente universitaria y crisis sanitaria COVID-19. CienciAmérica, 9 (2). http://dx.doi.org/10.33210/ca.v9i2.299

Urzúa, A., Vera, P., Caqueo, A. y Polanco, R. (2020). La Psicología en la prevención y manejo del COVID-19. Aportes desde la evidencia inicial. Terapia Psicológica, 38, (1). http://dx.doi.org/10.4067/s0718-48082020000100103

Venegas, L., Luzardo, H. J. y Pereira, A. (2020). Conocimiento, formación y uso de herramientas TIC aplicadas a la Educación Superior por el profesorado de la Universidad Miguel de Cervantes. Edutec. Revista Electrónica de Tecnología Educativa, (71), 35-52. https://doi.org/10.21556/edutec.2020.71.1405

Voogt, J. M., Almekinders, M., Van den Akker, J. J. H. y Moonen, B. (2005). A blended in-service arrangement for Classroom technology integration: Impacts on teachers and students. Computers in Human Behavior, 21, 523-539. https://doi.org/10.1016/j.chb.2004.10.003

Westbroek, H., De Vries, B., Walraven, A., Handelzalts A. y McKenney, S. (2019). Teachers as codesigners: Scientific and colloquial evidence on teacher professional. Development and curriculum innovation. En Pieters, J., Voogt, J., Pareja, N. (Eds.). Collaborative Curriculum Design for Sustainable Innovation and Teacher Learning. (35-54). Springer. https://link.springer.com/book/10.1007\%2F978-3-030-20062-6

\section{Para citar este artículo:}

Colorado Aguilar, B. L. (2020). Co-diseño del curso TIACE en la formación inicial docente de educación preescolar ante la contingencia de la COVID-19. Edutec. Revista Electrónica De Tecnología Educativa, (74), 170-189. https://doi.org/10.21556/edutec.2020.74.1815 